\title{
Essai de promotion et de défense d'un intérêt économique écossais par le gouvernement SNP
}

The SNP Government's Attempts to Promote and Defend Scottish Economic Interests

Edwige Camp-Pietrain

\section{(2) OpenEdition}

\section{Journals}

Édition électronique

URL : https://journals.openedition.org/etudesecossaises/411

DOI : 10.4000/etudesecossaises.411

ISSN : 1969-6337

Éditeur

UGA Éditions/Université Grenoble Alpes

Édition imprimée

Date de publication : 31 mars 2011

Pagination : 197-215

ISBN : 978-2-84310-191-5

ISSN : $1240-1439$

Référence électronique

Edwige Camp-Pietrain, « Essai de promotion et de défense d'un intérêt économique écossais par le gouvernement SNP », Études écossaises [En ligne], 14 | 2011, mis en ligne le 31 mars 2012, consulté le 12 avril 2023. URL : http://journals.openedition.org/etudesecossaises/411 ; DOI : https://doi.org/ 10.4000/etudesecossaises.411 


\section{Essai de promotion et de défense d'un intérêt économique écossais par le gouvernement SNP}

L'économie occupe une place prééminente dans le programme du Scottish National Party (SNP). Celui-ci prétend en effet que l'Écosse dispose de ressources adéquates pour assurer son autosuffisance. Ce postulat fut conforté par l'exploitation du pétrole de la mer du Nord à partir des années 1970. Le SNP ajoute que l'Écosse se trouvant privée de ses richesses qui abondent le budget britannique, elle paraît assistée et dépendante du reste de l'Angleterre (Levy, 1990, chap. 3). C'est ainsi que le SNP conclut à la nécessité de l'accession à l'indépendance.

Depuis une vingtaine d'années, le SNP essaie d'affiner ce raisonnement en fonction du contexte. La conquête du pouvoir impliquant une victoire aux dépens du parti dominant, travailliste, le SNP présentait un projet social-démocrate de redistribution (Gallagher, 1991, chap. 7). L'intérêt de l'Écosse consistait alors à répartir la manne pétrolière, tout en adhérant à la CEE pour éviter l'isolement. Cependant, la dévolution du pouvoir en 1999 l'amena à ajuster son programme, notamment en direction des entreprises. L'Écosse avait alors intérêt à attirer les investisseurs, tout en conservant une orientation social-démocrate. Les deux premières années de l'exercice du pouvoir, de 2007 à 2009, dans un contexte de crise économique et financière, exigèrent de nouveaux aménagements, qui relevaient davantage de la défense que de la promotion de l'Écosse.

Ce sont ces aspects que nous allons envisager, autour du personnage central que constitue Alex Salmond, ancien économiste au Scottish Office et à la Royal Bank of Scotland, mais aussi leader du parti de 1990 à 2000, puis à nouveau depuis 2004, député à Westminster depuis 1987, député à Holyrood de 1999 à 2001, puis à nouveau depuis 2007, et First Minister du gouvernement écossais depuis 2007. La perspective sera chronologique, mais nous nous interrogerons sur la nature de l'intérêt écossais défini par le SNP, notamment quant au rôle de la puissance publique et à la perception de l'Écosse en tant que victime ou responsable. 


\section{Le SNP dans les années 1990 : un parti redistributeur, en opposition au pouvoir central}

Avant la création d'un Parlement écossais décentralisé, le SNP avait pour objectif d'obtenir une majorité des 72 sièges écossais à la Chambre des communes, ce qui lui confèrerait un mandat pour négocier l'indépendance (SNP, 1997, p. 9). Alex Salmond, devenu leader du parti en 1990, s'attacha à renforcer la crédibilité de ses propositions, notamment au plan économique. Lui-même avait fait partie du 79 Group, qui, à l'intérieur du SNP, se voulait socialiste et partisan de l'action directe, ce qui le conduisit à occuper des sites industriels menacés de fermeture au début des années 1980. Au-delà, cette faction, vite bannie par la direction de l'époque, avait contribué à diffuser l'image d'un parti situé à gauche, alors que son programme, social-démocrate depuis le milieu des années 1970 (Wilson, 2009, p. 120), n'était pas ouvertement assumé jusque-là.

Alex Salmond souhaitait que son parti démente les critiques, en particulier celle des gouvernements britanniques, notamment conservateurs, selon lesquelles l'Écosse était subventionnée par le reste du RoyaumeUni, car les dépenses publiques excédaient les recettes locales. Salmond voulait aussi un parti en mesure de détailler ses propositions. Il renforça l'organisation, consolida le financement du SNP qui se dota d'un responsable des affaires économiques, Andrew Wilson, et commanda diverses études, ce qui lui permit d'établir des estimations chiffrées de la richesse intrinsèque de l'Écosse (Lynch, 2002, p. 209).

C'est ainsi que, pendant la campagne pour les élections législatives de 1992, le SNP publia un Manifeste économique, suivi du budget d'une Écosse indépendante l'année suivante (SNP, 1992; SNP, 1993). Il prévoyait de confier au nouvel État écossais un rôle redistributeur, s'agissant des dépenses sociales, mais aussi de l'investissement. Il créerait un fonds de participation dans les entreprises. Il s'engageait à nationaliser les actifs écossais de British Steel, au moment où l'entreprise venait d'annoncer la fermeture de son principal complexe écossais, Ravenscraig.

Afin de financer ces dépenses, le parti comptait sur les ressources pétrolières, qui ne seraient pas gaspillées, car une partie des impôts acquittés par les multinationales du pétrole serait placée dans un fonds souverain pour les générations futures, la fiscalité sur les entreprises demeurant inchangée. Quant aux particuliers, le parti préconisait une imposition plus progressive. À cet effet, il envisageait une baisse de l'impôt sur les revenus les plus faibles, mais une hausse des prélèvements pour les plus fortunés (suppression des dégrèvements, déplafonnement des cotisations). Il proposait néanmoins un relèvement des impôts indirects. Parmi les autres recettes escomptées, le parti prévoyait de réaliser des économies 
substantielles, notamment sur la défense et le nucléaire. Or ces ressources étaient particulièrement incertaines, résultats d'économies hypothétiques ou de revenus pétroliers fluctuants au gré des cours mondiaux.

Le SNP affirmait que le budget écossais serait d'abord légèrement déficitaire, puis excédentaire sur le moyen terme, tandis que les observateurs les plus optimistes tablaient sur un déficit, de l'ordre de 5 à $7 \%$ du PIB en 1992 (contre 4-5\% pour l'État britannique, ce qui ne paraissait pas insoutenable). Le SNP proposait une réponse aux attentes de la population écossaise qui rejetait le néolibéralisme des gouvernements conservateurs et qui semblait accepter des hausses d'impôts permettant d'améliorer les services publics et de défendre les emplois. Cependant, il n'avait aucune influence sur les décisions du gouvernement, en dépit de coups d'éclats ponctuels à la Chambre des communes, tel celui de Salmond qui, jeune député en 1988, s'éleva contre un budget incluant un impôt inique, la poll tax, ce qui lui valut une exclusion (Torrance, 2010, p. 94).

À la fin des années 1990, sous le double effet de la mondialisation et de la dévolution, le SNP entreprit un réexamen de sa position.

\section{Le SNP face aux institutions dévolues de 1999 à 2007 : une volonté de libération des capacités de l'Écosse par l'intermédiaire de la dévolution}

À partir de 1999, le SNP, premier parti d'opposition au Parlement écossais, avait pour objectif de gagner une majorité de sièges au Parlement écossais pour réclamer l'indépendance. En 1999, il évoquait encore la théorie du mandat, tandis qu'en 2003, il dissociait clairement le vote en sa faveur et l'indépendance, soumise à référendum (SNP, 1999, «Introduction»; 2003, p. 1). En conséquence, il devait démontrer sa crédibilité à gouverner les nouvelles institutions en précisant ses positions et en cessant de se définir constamment par rapport à Londres. Cette tâche fut accomplie sous la direction d'Alex Salmond et de John Swinney, son adjoint, ancien salarié d'une société écossaise d'assurance-vie, devenu leader du parti de 2000 à 2004, avant d'être chargé par Salmond des questions économiques. À ce noyau dur s'ajoutaient Andrew Wilson, désormais député au Parlement écossais, et Jim Mather, ancien chef d'entreprise nommé trésorier du parti.

Tout d'abord, en ce qui concerne la question fiscale, il faut distinguer les impôts sur les entreprises et sur les particuliers. Pour les premières, dès 1999, le SNP s'engagea à réduire la taxe professionnelle (business rates) et promit en 2003 un taux inférieur à celui pratiqué en Angleterre (SNP, 
1999, p. 9; SNP, 2003, p. 4). De plus, il envisageait une diminution de l'impôt sur les sociétés (corporation tax), impossible dans le cadre juridique de la dévolution, dont il prônait l'évolution. En conséquence, le SNP préconisait un abaissement de la pression fiscale sur les entreprises, afin de créer un avantage comparatif incitant les investisseurs à privilégier l'Écosse. Sa volonté d'accroître les pouvoirs fiscaux du Parlement écossais visait à rendre ce dernier responsable de ses décisions, l'un des objectifs de la dévolution.

Quant à la fiscalité sur les particuliers, en 1999, lors des premières élections au Parlement écossais, seul le SNP proposa d'utiliser le pouvoir fiscal extrêmement limité de ce dernier, afin d'investir dans les services publics écossais pour restaurer leur caractère public (SNP, 1999, p. 5). C'est ainsi qu'il axa sa campagne sur une hausse de $1 \%$ de l'impôt sur le revenu, qualifiée de «penny for Scotland». Plus exactement, il s'engagea à ne pas appliquer la baisse de $1 \%$ décidée par le gouvernement Blair au plan britannique. Cette promesse, hâtive, ne rencontra pas le succès escompté. Les Écossais préférèrent le Parti travailliste qui, ayant promis de ne pas faire usage du pouvoir fiscal du Parlement écossais, saisit immédiatement l'occasion de présenter le SNP comme le parti dépensier. En conséquence, par la suite, il ne fut plus question d'augmenter la pression fiscale, ce qui correspondait aux aspirations de Salmond. Quant à la taxe d'habitation liée à la valeur du logement (council tax), le SNP proposa de la remplacer par un impôt fondé sur les capacités contributives (local income tax). Il retrouva par ce biais des accents redistributeurs, notamment à partir de 2005, afin de renouer avec un impôt progressif (SNP, 2005, p. 12).

Ensuite, le SNP s'engagea à préserver le caractère public des services publics. Cela passait par des recrutements massifs dans les domaines de la santé, de l'éducation. Cela impliquait aussi l'extension de leur gratuité (droits universitaires, frais de soins personnels des personnes âgées, ou repas scolaires). Il exerça des pressions sur l'exécutif, qui aboutirent lorsque la proposition était soutenue par les libéraux-démocrates, partenaires minoritaires mais influents de la coalition au pouvoir. De plus, le programme nationaliste critiquait l'usage de capitaux privés, que les dirigeants écossais refusaient d'utiliser pour la gestion des services publics, mais qu'ils acceptaient pour la construction de bâtiments publics, sous la forme des public-private partnership (PPP). Le SNP promit d'abolir ceux-ci, en les remplaçant par un système interdisant à des entreprises privées de réaliser des bénéfices substantiels aux dépens du contribuable. Un trust de services publics émettrait des obligations afin de financer des investissements qu'il gèrerait au nom de la nation, avec pour seul objectif l'intérêt 
public. Ce projet fut constamment présenté à chaque élection à partir de 1999 (SNP, 1999, p. 6; 2003, p. 5).

Enfin, le SNP parvint à modifier son image, notamment grâce aux efforts de John Swinney (président de la Commission parlementaire des entreprises puis leader du parti). Auprès des particuliers, il réussit à se positionner en tant que défenseur de leurs intérêts, avec plus d'attention que les travaillistes écossais (par opposition au New Labour à Westminster, jugé plus indifférent) [Bromley et Curtice, 2003, p. 22]. La population nota son programme ambitieux de dépenses. Elle retint aussi l'image d'un parti très ambiguë, qui affichait certes une volonté de redistribution, mais qui, surtout, laissait entendre que ses réformes entraîneraient une réduction de la pression fiscale.

Le SNP se rapprocha aussi des milieux économiques et financiers, dont la confiance était nécessaire pour un parti ayant des ambitions gouvernementales. Les chefs d'entreprises, volontiers critiques à l'égard du principe de dévolution et d'hommes politiques écossais réputés enclins aux dépenses sociales, durent s'adapter au nouveau contexte. Ils cherchèrent à formaliser les contacts avec le Parlement écossais afin d'encourager ce dernier à promouvoir l'Écosse. Les travaillistes au pouvoir se montrant dans l'ensemble décevants (à l'exception notable de Wendy Alexander), le SNP sut répondre à leurs attentes. Les dirigeants nationalistes activèrent leurs réseaux et, dès 2002, ils procédèrent par rencontres successives avec des petits groupes de chefs d'entreprises, séduits par leurs promesses fiscales, leur renonciation à l'interventionnisme, leur analyse des faiblesses nationales (infrastructure, formation, démographie...), mais surtout par leur volonté de défendre les intérêts de l'Écosse.

La campagne précédant les troisièmes élections au Parlement écossais de 2007 marqua l'apogée de ces efforts (SNP, 2007; Camp, 2009). Le SNP obtint le soutien, verbal et financier, de poids lourds de l'économie écossaise, qu'il sut dévoiler tout au long de la campagne pour maintenir l'attention des médias tout en surprenant ses adversaires, jusqu'au gouvernement travailliste. Mettant en sourdine son objectif indépendantiste, grâce à la promesse d'un référendum qui renvoyait la question constitutionnelle à une date ultérieure, il s'engagea à appliquer son programme dans le cadre de la dévolution. Il voulait des impôts plus faibles et plus équitables. Ainsi, il abaisserait l'impôt sur les PME par l'intermédiaire de la taxe professionnelle existante, ainsi que l'impôt sur les sociétés s'il en avait le pouvoir. De même, il allègerait la taxe d'habitation, en transformant celle-ci en impôt local sur le revenu. En ce qui concerne les dépenses, il confirma son orientation social-démocrate. Il promit d'étendre la gratuité de nombreux services publics, en particulier l'enseignement supérieur, en abolissant les droits d'inscription forfaitaires 
écossais et en supprimant la dette des étudiants. Il maintint son projet de financement des investissements publics par un trust à but non lucratif, que les collectivités locales pourraient préférer au PPP. Malgré la contradiction évidente entre les deux volets de son programme, baisse des impôts et hausse des dépenses, Salmond balaya les objections, promettant de nombreuses économies dans le fonctionnement de l'administration, mais en évitant cette fois les chiffrages détaillés.

Les électeurs eux-mêmes appréciaient la perspective de baisses d'impôts et (quoique plus modestement) l'extension de l'universalité des services publics (Curtice et al., 2009, p. 93). Ils considéraient le SNP comme le plus à même de veiller aux intérêts écossais, même s'ils estimaient que l'économie était gérée à Londres (John et al., 2010, p. 64, 89 et 105). Cette stratégie permit aux nationalistes d'emporter, pour la première fois, une élection au plan écossais, et de former un gouvernement qui se fit fort de tenir ses engagements.

\section{Le SNP au pouvoir depuis 2007 : remises en cause défensives}

Le gouvernement dirigé par Salmond, assisté de Swinney aux finances et à la croissance durable, et Mather aux entreprises, se mit immédiatement au travail avec un objectif clair : démontrer sa compétence afin de convaincre une majorité d'Écossais d'accepter d'ouvrir des négociations sur l'indépendance de leur nation, proposition qu'il comptait leur soumettre par référendum avant la fin de la législature. L'économie constituait un axe majeur, car entreprises et particuliers devaient avoir confiance dans leurs capacités.

L'une des premières décisions de Salmond fut de s'entourer d'un conseil de conseillers économiques, se situant dans la filiation du conseil du président Truman en 1946. Parmi ses membres, se trouvaient d'anciens dirigeants d'entreprises connus (George Mathewson, son président, ancien PDG de la Royal Bank of Scotland et ancien directeur général de la Scottish Development Agency; Crawford Beveridge, ancien directeur général de Sun Microsystems et de Scottish Entreprise; Robert Smith, PDG du groupe Weir; Jim McColl, PDG de Clyde Bowers), des journalistes spécialisés (Frances Cairncross, The Economist), des conseillers d'organismes internationaux (Andrew Hughes Hallett, expert auprès du FMI et de la Banque mondiale), des universitaires (John Kay et un spécialiste du pétrole, Alex Kemp), une chercheuse irlandaise (Frances Ruane) et deux prix Nobel (Finn Kydland et James Murlees). Les deux premiers avaient appuyé le parti pendant la campagne. Aucun ne représentait explicitement les intérêts des salariés. Salmond expliqua que ce conseil, 
qui se réunirait une fois par trimestre, publierait un rapport annuel avec des recommandations (SP, 2007b, col. 1327).

À l'automne 2007, le gouvernement élabora une stratégie économique fixant des objectifs (rattraper la croissance du Royaume-Uni d'ici 2011 et celle des petits États européens à plus long terme) et désignant des modèles présentés comme l'«arc de prospérité» européen, qu'il s'agisse de la Norvège, pour son fonds souverain, ou de l'Islande ou l'Irlande, qui avaient su attirer les entreprises grâce à leur fiscalité (Scottish Government, 2007, p. 11). Il occultait la forte pression fiscale dans la première, qui rendait les deux exemples peu compatibles (Keating, 2009, p. 116).

En outre, dans son premier projet de loi budgétaire, le SNP abaissa la taxe professionnelle pour les PME, et la supprima pour les plus petites. Sous la pression des députés conservateurs, il accepta d'appliquer rapidement cette réforme. En échange de cette promesse, il obtint leur soutien, en janvier 2008, ce qui lui permit de faire approuver son budget par le Parlement écossais et, par conséquent, de se maintenir au pouvoir. Pour cette même raison, les travaillistes se réfugièrent dans l'abstention, en s'érigeant en garants de la social-démocratie écossaise mise à mal par ces baisses de recettes (SP, 2008a, col. 5864, Ian Gray).

À partir de là, le gouvernement nationaliste perdit la maîtrise du calendrier, subissant trois types de contraintes : l'arithmétique parlementaire, les limites juridiques de la dévolution et la crise économique. Il dut se contenter de réagir à des facteurs indépendants de sa volonté, en particulier avec la crise financière. Mais Salmond tenta d'exploiter chaque contretemps.

En ce qui concerne les politiques emblématiques qu'il envisageait en 2007, les avancées sont inégales. Tout d'abord, les députés nationalistes, minoritaires, devaient trouver des alliés lors de chaque vote. Or aucun de leurs adversaires n'a soutenu la création d'un impôt local sur le revenu. Seuls les libéraux-démocrates étaient favorables à ce projet, mais à condition de laisser chaque collectivité en fixer le montant, alors que le SNP voulait maintenir un taux unique dans toute l'Écosse. L'opposition dénonçait la perspective de hausse de la pression fiscale pour la plupart des contribuables. De plus, les milieux d'affaires rejetaient cet impôt qui alourdirait les obligations des entreprises (retenue à la source) et les charges des plus petites (tenues de l'acquitter), alors que le gouvernement écossais préférait éviter de les aliéner. Quant au gouvernement britannique, il prévoyait de supprimer l'allocation versée aux ménages les plus démunis (housing benefit) pour leur permettre de payer leur taxe d'habitation, ce qui remettait en cause l'équilibre financier du dispositif. En février 2009, Swinney renonça à cette réforme parce qu'il avait entendu la position majoritaire au Parlement, d'autant que Mather était réservé 
(Donald, 2009). Il s'est contenté d'exercer des pressions sur les conseils des collectivités locales pour éviter toute hausse de la taxe d'habitation existante.

Ensuite, le dispositif imaginé par le gouvernement nationaliste pour supplanter les PPP s'est heurté non à la composition du Parlement (il ne nécessitait pas de loi), mais au cadre juridique de la dévolution. Il reposait sur l'émission d'obligations par un trust, comme l'annonça John Swinney en décembre 2008. Or le Parlement écossais n’était pas autorisé à emprunter sauf sur le très court terme, avec l'accord du secrétaire d'État à l'Écosse. À défaut, le gouvernement écossais a proposé de confier cette tâche aux collectivités locales, qui, elles, en avaient le droit. Elles devraient former des consortiums avec les conseils de santé et autres organismes publics, qui, groupés, pourraient imposer des taux d'intérêt plus faibles. Leurs investissements, de faible envergure, seraient coordonnés (mais non supervisés) par un Scottish Futures Trust. En septembre 2008, Salmond sut créer l'événement en désignant le président de cet organisme, Angus Grossart, fondateur de la banque d'affaires Noble Grossart (Dinwoodie, 2008a). L'expérience et les réseaux de ce grand nom de la finance écossaise devaient permettre de convaincre les sociétés privées d'investir, tout en négociant une meilleure utilisation de l'argent public. Il était question de restreindre les bénéfices privés, et non plus de les prohiber. Or ce trust n'a pas précisé ses modalités d'action, se contentant d'encourager quelques pratiques collectives locales. Dans l'intervalle, de nombreux projets de constructions et rénovations d'écoles et autres bâtiments publics étaient en attente. Les milieux d'affaires, tout comme les syndicats, s'en sont indignés. L'opposition parlementaire estima que ce projet amendé demeurait proche des PPP. Elle incitait le gouvernement à autoriser les collectivités locales à choisir leur mode de financement, comme il s'y était engagé jusque-là (SP, 2009d, col. 16378, Gavin Brown, conservateur; col. 16387, Jamie Stone, liberal-démocrate; col. 16394, David Whitton, travailliste).

Salmond et Swinney n'ont pas manqué de souligner que ces contraintes disparaîtraient si le Parlement écossais se voyait conférer des pouvoirs supplémentaires, notamment celui d'emprunter. Ils avaient sur ce point le soutien des libéraux-démocrates (SP, 2009c, col. 14724, Tavish Scott). De même, ces pouvoirs étaient prônés par la commission Calman, chargée par le gouvernement britannique et le Parlement écossais de réviser le Scotland Act de 1998 (Commission on Scottish Devolution, 2009, chap. 3 et 5). Mais le gouvernement britannique expliqua qu'il ne pouvait sélectionner parmi les recommandations de celle-ci, même pour un projet ponctuel comme le nouveau pont sur la rivière Forth (Dinwoodie, 2009b). De plus, en public, le SNP préférait ignorer les probables diffi- 
cultés à emprunter d'une nouvelle institution, notamment la nécessité de proposer un taux d'intérêt plus rémunérateur (Jones, 2002, p. 247).

Enfin, le budget écossais était quasi exclusivement alimenté par une dotation du gouvernement britannique. Or à l'été 2007, après une décennie d'envolée des dépenses publiques, Gordon Brown, devenu Premier ministre, engagea une révision liée à la conjoncture. En conséquence, le budget écossais, qui avait bénéficié de ces largesses grâce à la formule Barnett, connaîtrait une moindre progression, obligeant le gouvernement écossais à renoncer à ses promesses d'extension de la gratuité des services publics. Par exemple, si celui-ci a supprimé les droits universitaires, il a simplement réduit la dette des étudiants, faute de pouvoir l'annuler. Il devait abolir le ticket modérateur par étapes.

Le budget pour l'année 2009 et les perspectives pour les années suivantes étaient encore plus sombres dans le contexte de crise économique. Salmond s'est insurgé contre les effets sur la formule Barnett des économies imposées par le gouvernement britannique aux services publics anglais. Jim Murphy, secrétaire d'État à l'Écosse, les chiffra à 367 millions sur un budget écossais de 30 milliards, tandis que Salmond et Swinney ne cessèrent d'avancer la somme de 500 millions (jugée équivalente à 9000 emplois publics), car ils ajoutaient les crédits «perdus» par l'Écosse en raison de la réduction des dépenses publiques de santé en Angleterre (Crichton, 2009).

Salmond dénonça une baisse de la dotation écossaise, alors que c'est sa progression qui devait ralentir. Il réclama des compensations. Ayant fait usage des crédits non utilisés les années antérieures, il avait peu de marge de manœuvre. Il suggéra au gouvernement britannique de renoncer à certaines politiques réservées (comme le nucléaire), libérant ainsi des fonds pour les services publics anglais, dont l'Écosse bénéficierait grâce à la formule Barnett. Il demanda, sans succès, l'accès à des fonds écossais détenus par des organismes britanniques. Il exigea, en vain, le rétablissement de l'allocation supprimée par le gouvernement britannique lorsque le Parlement écossais avait mis en place la gratuité des soins personnels aux personnes âgées (attendance allowance). En fait, il souhaitait le retour à un passé idéalisé, dans lequel l'Écosse retrouverait des fonds qui, bien que publics, lui étaient dus. Ses députés évoquèrent un rapatriement, de droit, lors des débats sur la prise en charge des frais des personnes âgées (SP, 2007a, col. 819, Ian McKee; SP, 2008b, col. 8742, Shona Robison). Ainsi, l'Écosse serait dispensée de participer à l'effort collectif de modération des dépenses publiques. Une fois encore, «Londres» était rendu responsable de la situation.

Refusant d'admettre publiquement que ces contraintes budgétaires pourraient l'amener à abroger certaines politiques existantes, le First 
Minister écossais affirma qu'il était empêché de mener une politique de relance. À défaut, la configuration du Parlement écossais conduisit Swinney, en janvier 2009, à donner des gages à chacun de ses adversaires pour faire voter son budget, dont la version initiale avait été rejetée en première lecture : hausse du nombre d'apprentis pour satisfaire les travaillistes, aide à la reconversion de l'activité en centre-ville pour contenter les conservateurs, création d'un groupe de travail sur la crise financière pour apaiser les libéraux-démocrates. Accusé de manquer de cohérence, Swinney se félicita d'appartenir à un gouvernement à l'écoute de la volonté des représentants du peuple (SP, 2009b, col. 14649).

Face à la crise financière, son plan d'action consistait surtout à renforcer les conseils à la création d'entreprises et à développer la formation, ce qui posait problème en raison des réorganisations structurelles en cours. La principale affectait Scottish Entreprise que le SNP voulait rationaliser : les LEC avaient été supprimées en mars 2008 (créant des difficultés pour les PME en milieu rural), l'information aux entreprises (business gateway) relevait désormais des collectivités locales, tandis que ses missions sociales avaient été confiées à une nouvelle structure, Skills Scotland. Elle devait se recentrer sur l'aide au développement des entreprises et sur la fourniture des infrastructures indispensables à des secteurs prioritaires comme l'énergie ou la finance (McConnell, 2009). Le gouvernement s'engageait aussi à encourager le tourisme (profitant du Homecoming de l'année 2009), intensifier les travaux publics (mais son opposition aux PPP freinait la progression) et à utiliser par anticipation des fonds européens (fonds de développement régional et fonds social).

Le gouvernement écossais assura agir au mieux, dans la limite de ses compétences. Le premier rapport annuel du conseil économique d'Alex Salmond, publié en décembre 2008, le conforta dans son action. Il comportait peu de propositions embarrassantes, sauf peut-être la recommandation d'examen de toutes les sources d'énergie, nucléaire inclus, que Swinney accepta de soumettre à enquête (SP, 2009a, col. 14296).

Dans tous les cas, Salmond refusait de relancer par la baisse de l'impôt sur le revenu comme le proposaient les libéraux-démocrates au moment de la discussion du budget, et comme l'envisageait désormais Tom Hunter, un des chefs d'entreprise qui l'avait soutenu. Il expliqua que l'Écosse ne pouvait se le permettre. Mais il écartait aussi toute hausse d'impôts pour compenser la moindre progression de la dotation britannique, comme le suggérait Hallett, membre de son conseil. Alors qu'il prônait un accroissement des pouvoirs fiscaux du Parlement écossais, il n'était pas encore prêt à faire usage de l'intégralité de ceux dont il disposait. Un autre de ses conseillers, Kay, fustigeait quant à lui le coût prohibitif de la collecte. 
S'agissant plus précisément de la crise financière, elle a fortement affecté l'Écosse, laissant le gouvernement écossais relativement impuissant. À partir de septembre 2008, les deux grandes banques écossaises ont été au cœur de celle-ci, en raison de leur croissance démesurée dans les crédits à risque. La Bank of Scotland, intégrée à HBOS depuis sa fusion avec Halifax en 2001, et la Royal Bank of Scotland, rencontrèrent des difficultés de refinancement avant d'afficher des pertes colossales. C'est le gouvernement britannique qui imposa à HBOS de fusionner avec Lloyds, avant de prendre des participations croissantes dans le groupe issu de cette fusion, et dans la Royal Bank of Scotland, tout en garantissant les dépôts. De même, en mars 2009, la mutuelle écossaise Dunfermline Building Society fut dans l'incapacité de faire face à ses engagements, victime de ses investissements à risque et de la concurrence agressive des banques écossaises recapitalisées. Le gouvernement britannique ordonna sa reprise par une autre société mutuelle, Nationwide, en lui versant des compensations, tandis que la Banque d'Angleterre se chargeait des actifs à risque.

Alex Salmond et son gouvernement se trouvèrent marginalisés, les compétences économiques et financières étant réservées au gouvernement britannique, alors que la finance représentait un secteur important de l'économie écossaise : 8\% de la richesse, $9 \%$ des emplois lui étaient directement liés, sans compter les emplois indirects. Pire, Salmond sembla perdre quelque peu son habileté tactique. Lui qui, pendant la campagne électorale de 2007, avait loué la politique de faible réglementation des marchés financiers et salué la probité particulière des financiers écossais, éprouva des difficultés d'adaptation (McWhirter, 2009).

Or Salmond conserva son admiration et sa confiance envers les financiers écossais, même si depuis le début de la décennie, des documents internes au parti mettaient en garde contre leurs pratiques (Cuthbert et al., 2009, p. 116). Il commença par blâmer quelques spéculateurs pratiquant la vente à découvert, feignant d'ignorer que l'ensemble des pratiques bancaires était en cause (SP, 2008c, col. 11109, Wendy Alexander, travailliste). De même, il récusa l'idée que la direction de DBS ait pu s'engager dans des investissements à risque aux États-Unis. De plus, parmi son conseil économique se trouvait ce type de spéculateurs, notamment son président George Mathewson, dirigeant d'un fonds spéculatif enregistré dans un paradis fiscal, sans état d'âme à l'égard des bonus, ou Jim McColl, domicilié à Monaco (SP, 2008c, col. 11122, David Whitton, travailliste et col. 11124, Jeremy Purvis, liberal-démocrate; Hutcheon, 2009a; Hutcheon, 2009b). Salmond rétorqua que Mathewson, s'il était responsable de la transformation de la Royal Bank of Scotland en entreprise internationale, n'était pas entaché par les événements récents (Paterson, 
2009). L'opposition rappela qu'il avait nommé Goodwin directeur général de la banque (SP, 2009b, Murdo Fraser, conservateur). Seule une députée nationaliste d'Édimbourg, Shirley-Anne Sommerville, s'en prit à l'ancienne direction de l'établissement financier. Le leader du SNP justifia également le coût exorbitant des réunions de son conseil (Hutcheon, Gordon, 2009). Mais en septembre 2009, il admit implicitement les excès, en utilisant les bâtiments publics existants pour les réunions au lieu de louer des demeures somptueuses.

En outre, Salmond ne manqua pas une occasion de manifester son aversion à l'égard de solutions imposées par Londres, quasiment vécues comme une conspiration contre le secteur financier écossais. Il retrouva la stratégie du bouc émissaire. Il interpréta la fusion de HBOS et Lloyds comme une absorption de la banque écossaise, pourtant présentée comme indispensable à la survie de l'entreprise. Il aurait préféré une recapitalisation publique de chacune des banques. S'il obtint le soutien des libérauxdémocrates (SP, 2008d, Tavish Scott), le gouvernement britannique lui opposa une fin de non-recevoir. Le député nationaliste Alex Neil appuya la tentative de quelques financiers écossais, dont Mathewson et Peter Burt, appelant les actionnaires à rejeter la fusion, en vain. Ils avaient néanmoins tous deux contribué à créer cette «bulle financière». Neil soutint également la recherche de capitaux écossais pour renflouer la banque (Dinwoodie, 2008b). Par ailleurs, les députés nationalistes à la Chambre des communes essayèrent de contrer la décision du ministre britannique des entreprises de s'affranchir des lois protégeant la concurrence, sans succès. Puis des dirigeants économiques écossais formèrent un Merger Action Group, contre cette résolution, mais ils furent déboutés par les tribunaux.

De même, Salmond aurait voulu garder une mutuelle écossaise indépendante afin de défendre une société écossaise engagée dans le financement des bailleurs sociaux privés (SP, 2009d, col. 13658). Il avait d'ailleurs proposé une prise de participation de 25 millions de livres par son gouvernement, en complément de celle d'autres investisseurs, voire de l'État britannique. Or non seulement ce dernier lui refusa cet investissement, mais qui plus est, il déboursa 1,6 milliard de livres pour assurer la survie de la mutuelle. Salmond dut admettre qu'il n'avait pas accès à toutes les données.

En outre, cette crise sembla décrédibiliser le projet économique du SNP, axe central de son objectif indépendantiste. Salmond proclama qu'un gouvernement indépendant aurait sauvé les deux grandes banques en tant qu'entités écossaises. Or il aurait été incapable de financer leur sauvetage : les 100 milliards de livres qu'il évoquait représentaient trois fois le budget écossais, mais six fois moins que la somme investie par le 
gouvernement britannique. Ce projet impliquait l'existence d'une banque centrale écossaise qui ne figurait pas dans son programme. Or cette création nécessiterait des compétences particulières et la confiance des déposants, Écossais ou Anglais (Jones, 2002, p. 246-248). Quant à l'Arc de prospérité, il s'enfonça dans de graves difficultés dès le début de la crise, l'État islandais étant en faillite, tandis que l'Irlande peinait à soutenir ses banques et à conserver les investissements étrangers. Salmond, rappelant le caractère mondial de la crise, loua néanmoins la rapidité de la réaction du gouvernement irlandais (MacNab, 2008). Les députés travaillistes et conservateurs martelèrent que seule l'Union permettait la survie des fleurons de la finance écossaise (SP, 2008c, col. 11095, Iain Gray, travailliste et col. 11099, Annabel Goldie, conservateur).

Salmond et Swinney durent se contenter de quatre débats au Parlement écossais, purement consultatifs. À la fin du mois d'avril 2009, celui-ci envisageait, sur proposition de Margo MacDonald (ancienne députée SNP, désormais indépendante), une commission d'enquête, mais il devait définir un cadre qui n'empiète pas sur les compétences réservées à Westminster (SP, 2009e, col. 17400). Ce projet n'avait pas les faveurs de certains conseillers de Salmond, tel Mathewson, qui préférait se cantonner à l'enquête diligentée par les autorités financières britanniques (Hutchinson, Gordon, 2009).

Salmond dut se résoudre à admettre que la crise financière avait un impact considérable sur l'économie réelle. Même si son gouvernement souligna que le taux de chômage écossais au premier trimestre 2009 $(5,4 \%)$ était inférieur à la moyenne britannique $(6,7 \%)$, selon l'estimation de DT2 pour Scottish Enterprise, 24000 emplois liés au secteur de la finance étaient menacés d'ici 2011 (Donnelly, 2009). Salmond et Swinney organisèrent des rencontres avec les nouveaux dirigeants des banques pour leur demander de préserver l'emploi en Écosse et notamment les fonctions de direction. Ils mirent en place une task force sur les services financiers, en partenariat avec les milieux financiers et les syndicats, comme les y invitaient les libéraux-démocrates (SP, 2009c, col. 14784, Jeremy Purvis). Conformément à la volonté des partenaires sociaux, le gouvernement écossais envisageait la création d'une banque écossaise d'investissement, financée par le budget écossais et les fonds européens de développement, afin de soutenir les firmes innovantes.

Cela n'empêcha pas le groupe Lloyds d'annoncer en juillet la suppression de plus de 200 emplois en Écosse. De plus, le nouvel interventionnisme de Salmond déplaisait à CBI Scotland, comme le fit valoir son directeur lors de l'annonce par Diageo de la fermeture d'usines d'embouteillages du whisky en Écosse à l'été 2009 (Young, 2009). Il était également récusé par la direction de la société qui rejeta le plan de sauvetage 
élaboré par le groupe de travail multipartite présidé par Swinney, car il méconnaissait les règles économiques élémentaires (Currie, 2009).

Ainsi, le gouvernement écossais, seul, avait peu de pouvoirs. Par conséquent, après avoir tenté de donner des conseils au gouvernement britannique, Salmond manifesta sa volonté de coopération pour défendre les emplois écossais. Jim Murphy, nommé secrétaire d'État à l'Écosse à temps plein en octobre 2008, se déclara lui aussi partisan du dialogue. Les deux exécutifs se disputaient la paternité des annonces, comme lors du sommet économique en mars, tout en évitant de se critiquer. Le gouvernement écossais s'associa à la mise en œuvre des projets britanniques, comme l'accompagnement des chômeurs (la formation étant dévolue) ou le plan en faveur de la réduction des émissions de $\mathrm{CO}_{2}$. Néanmoins, Gordon Brown refusa obstinément de placer gouvernements écossais et britannique sur un pied d'égalité. Au printemps 2009, lors de la réunion de son Cabinet en Écosse, il n'accepta qu'une rencontre privée avec les leaders des partis politiques. Le sommet des forces économiques prévu d'ici fin 2009 devait associer Murphy, Salmond et les partenaires sociaux.

Dans certains cas, existaient des politiques concurrentes, notamment dans la prévention de la saisie de maisons. Les Anglais ayant exigé des créanciers qu'ils n'y aient recours qu'en dernier ressort, tous les partis d'opposition prônaient l'extension de ce dispositif à l'Écosse. Le gouvernement SNP se prévalait d'une loi écossaise de 2001, jusque-là jugée plus protectrice car elle autorisait les propriétaires en difficultés à se tourner vers la justice. Or elle nécessitait une démarche volontaire. Le gouvernement ajoutait qu'ils pouvaient solliciter une prise de participation publique dans leur logement. Mais en 2009, il fixa un plafond pour encadrer le déclenchement de ce type d'action, qui, à 100000 de livres, était modeste par rapport au marché.

Au-delà, Salmond souhaitait retrouver une influence, après avoir été éclipsé par Gordon Brown. Il rencontra le Lord Mayor de la City de Londres, visita la Chine en avril pour promouvoir les universités écossaises, le whisky, le golf, les énergies renouvelables et le secteur financier écossais. Il envisageait un sommet d'économistes internationaux pour célébrer Adam Smith. De son côté, Jim Mather se rendit aux États-Unis (premiers investisseurs étrangers en Écosse), dans le cadre de la semaine de l'Écosse, pour rencontrer des investisseurs potentiels et conforter la réputation de son pays. Quant à Swinney, il se tourna vers le Japon, afin d'attirer investisseurs et consommateurs. 


\section{Conclusion}

Alex Salmond, fin stratège dans l'opposition, parvint à conquérir le pouvoir en profitant de l'impopularité du gouvernement britannique travailliste. En 2009, il devait composer avec la tactique qui lui avait permis de gagner les élections : volonté de distribution sans se soucier réellement des recettes, confiance dans le secteur économique et financier écossais. Le gouvernement SNP n'était pas redistributeur. Il assurait la distribution de ressources en voie de diminution. Il cherchait certes à défendre un intérêt écossais, mais un intérêt fort coûteux, exigeant une envolée des dépenses. Grâce à cette stratégie, il s'est posé en représentant des attentes du peuple face à un Parti travailliste dont l'action avait été trop limitée. Cela passait par une extension de la gratuité des services publics et une hausse des dépenses publiques. Salmond voulait également réduire les impôts afin de stimuler l'activité et d'accroître les recettes. Mais sur le court terme, son raisonnement engendrait des déséquilibres, que ne parvenaient pas à compenser les économies de gestion qu'il avait promises. De plus, le contexte de crise accentua l'écart entre ressources et dépenses, obligeant le SNP à renoncer à certaines promesses. En conséquence, Salmond retrouva ses accusations traditionnelles à l'encontre de Londres, responsable de tous les maux et principale source d'obstruction à l'action des représentants du peuple écossais. L'Écosse aurait des droits à l'intérieur du Royaume-Uni, bafoués par le gouvernement britannique. Elle s'était de surcroît vue privée des fleurons de son secteur financier par les nationalisations.

Or, début 2009, Salmond conservait une bonne image. Il demeurait plus populaire que ses adversaires, notamment pour son aptitude à défendre les intérêts écossais, et cette vision était partagée par ses électeurs, mais aussi par le plus grand nombre d'électeurs conservateurs et libérauxdémocrates (sondage YouGov commandé par le SNP, Dinwoodie, 2009a). Parallèlement, le sauvetage de banques écossaises par le gouvernement britannique renforçait le rejet de l'indépendance ou, au mieux, suscitait l'indifférence d'une opinion plutôt réticente à celle-ci (Curtice, 2009).

Pour la campagne aux élections européennes de 2009, les nationalistes ont choisi de mettre en avant les capacités de l'Écosse, non sans renoncer à présenter celle-ci comme une victime, car le nouveau projet de loi de finances du gouvernement britannique devait les contraindre à des réductions drastiques de dépenses (SNP, 2009, p. 1). Cette stratégie a permis au SNP de devancer les travaillistes de plus de 8 points de pourcentage. 


\section{Post-scriptum}

Lors des élections législatives à la Chambre des communes de mai 2010, Alex Salmond s'est clairement placé sur la défensive, clamant que seuls les députés nationalistes étaient capables de protéger l'Écosse des coupes budgétaires envisagées par le Parti conservateur, en tête dans les sondages d'opinion britanniques (SNP, 2010, p. 6-7). Il a souligné que celles-ci n'étaient pas inéluctables, à condition de supprimer les dépenses inutiles (cartes d'identité, Trident) et de traiter l'Écosse équitablement (compensation pour les investissements à Londres en prévision des Jeux olympiques, rétention du produit de la taxe sur les producteurs d'énergie fossiles). S'agissant des dépenses, il continuait à promettre la gratuité des services publics, ainsi que la création d'emplois grâce aux énergies renouvelables. Mais il n'a pu se prévaloir de son bilan, face à des travaillistes plus offensifs, affirmant la nécessité d'effectuer des choix, tout en dénonçant un gouvernement écossais qui avait délaissé les bastions industriels (abandon du projet de desserte ferroviaire rapide de l'aéroport de Glasgow). En ce qui concerne les recettes, Salmond maintenait sa volonté de réduire les prélèvements obligatoires (s'opposant au relèvement des cotisations prévu par le gouvernement sortant). Il devait veiller à ne pas paraitre trop proche des conservateurs qui venaient d'approuver son troisième budget (obtenant cette fois en échange une commission de surveillance des dépenses publiques et un soutien à l'investissement [SP, 2010a, col. 23364, John Swinney]). Il a notamment esquivé la possibilité de transformation de la distribution d'eau en mutuelle. Néanmoins, ce sont les conservateurs qui avaient les faveurs des milieux d'affaires, car ils étaient susceptibles d'exercer le pouvoir. Par ailleurs, au plan institutionnel, raison d'être du SNP, le message était brouillé, Salmond devant tacitement reconnaitre qu'il ne pourrait organiser de référendum sur l'ouverture de négociations menant à l'indépendance. Il promettait d'accroitre les compétences du Parlement écossais, au-delà de celles recommandées par Calman.

Cette stratégie s'est soldée par un échec. En dépit d'une progression de deux points, le SNP a conservé le même nombre de sièges qu'en 2005, soit 6 (perdant Glasgow East, emporté lors d'une élection partielle), loin de l'objectif ambitieux de Salmond (20), dont le programme et l'action étaient critiqués, tant au sein de l'électorat que dans son parti. La crise économique britannique intensifiait le décalage entre redistribution socialdémocrate et baisse des impôts, sans toutefois inciter les Écossais à se replier sur leurs intérêts étroits.

Après la formation par David Cameron du gouvernement de coalition, Salmond a fustigé son manque de légitimité, en vain car les travaillistes ont refusé de le cautionner. Le 30 novembre 2010, jour de la 
Saint-André, initialement envisagé pour la tenue du référendum sur l'indépendance, Michael Moore, secrétaire d'État (libéral-démocrate) à l'Écosse a publié un projet de loi reprenant des propositions de Calman, parmi lesquelles la dévolution de taxes supplémentaires (droit de timbre, taxe d'enfouissement des déchets), l'utilisation effective du pouvoir de variation de l'impôt sur le revenu et une autorisation limitée d'emprunt pour le Parlement écossais. Le gouvernement SNP a aussitôt rétorqué que ces pouvoirs, insuffisants, ne permettraient pas de relancer l'économie écossaise (SP, 2010b, col. 31004, Fiona Hyslop). De plus, il a dû avouer qu'il n'avait pas acquitté les charges annuelles exigées par le gouvernement britannique pour l'éventuelle modulation de l'impôt, ce qui obérait toute possibilité de mise en œuvre sur le court terme. Cette attitude défensive ne pourra toutefois être poursuivie, alors que le projet semble relativement consensuel.

\section{Références bibliographiques}

Bromley Catherine et Gurtice John, «Devolution: Scorecard and Prospects », dans C. Bromley, J. Curtice, K. Hinds et A. Park (éds), Devolution-Scottish Answers to Scottish Questions?, Édimbourg, Edinburgh University Press, 2003, p. 7-29.

Camp Edwige, "Les facteurs de la victoire du SNP aux élections au Parlement écossais de 2007 », Études écossaises, n 12, 2009, p. 170-184.

Commission on ScotTish Devolution, Serving Scotland Better, Édimbourg, 2009.

Crichton Torcuil, «Battle over What Budget Means for the People of Scotland», The Herald, 23 avril 2009, p. 20.

Currie Brian, «Salmond defends His Megaphone Diplomacy Over Diaego Closures», The Herald, 11 septembre 2009, p. 6.

Curtice John, «Public Attitudes and Elections», dans P. Cairney (éd.), Scottish Devolution Monitoring Report, Londres, Constitution Unit, janvier 2009, p. 17-29.

Gurtice John, MaCrone David, MaEwen Nicola, Marsh Michael et Ormston Rachel, Revolution or Evolution? The 2007 Scottish Elections, Édimbourg, Edinburgh University Press, 2009.

Cuthbert Jim et Guthbert Margaret, «SNP Economic Strategy. NeoLiberalism with a Heart», dans G. Hassan (éd.), The Modern SNP. From Protest to Power, Édimbourg, Edinburgh University Press, 2009, p. 105119.

Dinwoodie Robbie, «Salmond Brings in Top Financier to Run Public Investment Trust», The Herald, 11 septembre 2008, p. 1. 
-, «Cracks in Holyrood Consensus on HBOS», The Herald, 25 septembre 2008, p. 7.

—, «Salmond's Financial Competence at Heart of Poll Boost», The Herald, 25 avril 2009, p. 6.

—, «Treasury Refuses to Bend on Forth Bridge’s £2 bn Crossing», The Herald, 10 août 2009, p. 6.

Donald Colin, «The Speech Therapist», Sunday Herald, 15 février 2009.

Donnelly Brian, «Finance Sector to Lose 24,000 Scots Jobs by 2011, Says Study», The Herald, 13 avril 2009, p. 1.

Gallagher Tom, «The SNP and the Scottish Working Class», dans T. Gallagher (éd.), Nationalism in the Nineties, Édimbourg, Polygon, 1991, p. 102-125.

Hutcheon Paul, «Salmond and his Economic Adviser at Odds over Bank Bonuses», Sunday Herald, 22 février 2009.

—, «Salmond Hits Back in Row Tax Exile», Sunday Herald, 29 mars 2009.

Hutcheon Paul et Gordon Tom, «£100,000 Bill for Salmond's Aides», The Herald, 22 août 2009, p. 8.

Hutchinson Peter et Gordon Tom, «We Want Probe into Failed Banks », Sunday Herald, 19 avril 2009.

John Robert, Denver David, Mitchell James et Pattie Charles, Voting for a Scottish Government, Manchester, Manchester University Press, 2010.

Jones Peter, «The Economics of Independence», dans J. Murken, P. Jones Peter et M. Keating (éds), Scottish Independence, Edimbourg, Edinburgh University Press, 2002, p. 236-239.

Keating Michael, The Independence of Scotland, Oxford, Oxford University Press, 2009.

LEvy Roger, Scottish Nationalism at the Crossroads, Édimbourg, Scottish Academic Press, 1990.

Lynch Peter, The History of the SNP, Cardiff, Welsh Academic Press, 2002.

MaConnell Iain, «Scottish Entreprise Chief to Quit», The Herald, 30 mai 2009, p. 1.

MacNab Scott, «Nationalists' "Arc of Insolvency" Goals Attacked», The Herald, 13 octobre 2008, p. 6.

MaWhirter Iain, «The Curse of a Nation Once Renowned for Prudence», The Herald, 30 mars 2009, p. 16.

PAterson Stewart, «Salmond's Top Economic Adviser in Tax Haven Row», The Herald, 2 mars 2009, p. 7.

Scottish National Party (SNP), Recovery in Scotland: Make it Happen Now!, Édimbourg, 1992.

_, The Scottish Budget for an Independent Scotland, Édimbourg, 1993. 
—, Yes We Can Win the Best for Scotland, Édimbourg, 1997.

—, Scotland's Party, Scotland's Parliament, Édimbourg, 1999.

—, We Stand for Scotland, Édimbourg, 2001.

—, Release our Potential, Édimbourg, 2003.

—, Make Scotland Matter, Édimbourg, 2005.

—, It's Time, Édimbourg, 2007.

-, We've Got What it Takes, Édimbourg, 2009.

—, Elect a Local Champion, Édimbourg, 2010.

Scottish Government, The Government Economic Strategy, Édimbourg, 2007.

Scottish Parliament (SP), Official Report, 14 juin 2007.

—, Official Report, 28 juin 2007.

—, Official Report, 6 février 2008.

-, Official Report, 15 mai 2008.

-, Official Report, 24 septembre 2008.

—, Official Report, 30 octobre 2008.

—, Official Report, 22 janvier 2009.

—, Official Report, 4 février 2009.

—, Official Report, 5 février 2009.

-, Official Report, $1^{\text {er }}$ avril 2009.

—, Official Report, 13 mai 2009.

—, Official Report, 3 février 2010.

-, Official Report, $1^{\text {er }}$ décembre 2010.

Torrance David, Salmond: Against the Odds, Édimbourg, Birlinn, 2010.

WILson Gordon, SNP: the Turbulent Years 1960-1990, Stirling, Scots independent, 2009.

Young Alf, «We Have a Choice Between Open Market and State Control», The Herald, 31 juillet 2009, p. 17. 